\title{
Sonnets d'Yves Bonnefoy, Valérie Rouzeau et Robert Marteau : plan de texte et généricité
}

\author{
Monte, Michèle \\ Université de Toulon, BABEL EA 2649 \\ michele.monte@univ-tln.fr
}

Je partirai dans cette étude des propositions sur la structuration des textes présentées par Jean-Michel Adam dans La linguistique textuelle ( $3^{\mathrm{e}}$ édition) et de ses travaux sur la généricité, menés en collaboration avec Ute Heidmann. Je m'interrogerai tout d'abord sur le statut du sonnet : doit-on le définir comme un plan de texte ou comme un genre? Quelles sont les implications de l'une ou l'autre option? J'examinerai ensuite des œuvres récentes écrites en sonnets en montrant le parti qu'elles tirent de la forme choisie et les relations génériques complexes dans lesquelles elles sont prises et qui en éclairent la lecture ${ }^{1}$.

\section{Le sonnet : genre ou plan de texte ?}

Ainsi que le fait remarquer Schaeffer dans Textes et sens (1996: 49), la réflexion littéraire sur les genres s'accommode volontiers d'une hétérogénéité de critères. C'est pour cela que J.-M. Adam préfère inscrire sa réflexion dans le sillage de Bakhtine qui définit les "genres de la parole » comme des «types relativement stables d'énoncés » possédant des propriétés communes d'ordre thématique, compositionnel et stylistique parce qu'ils s'inscrivent dans le même domaine de l'activité humaine dont ils « reflètent les conditions spécifiques et les finalités » (1984:265, je cite d'après Adam 2011). C'est aussi le point de vue de Rastier qui écrit «Un discours s'articule en divers genres, qui correspondent à autant de pratiques sociales différenciées à l'intérieur d'un même champ. » (1989: 40). Rastier définit le genre comme une « interaction normée » entre des composantes du plan de l'expression et du plan du contenu (thématique, dialogique, dialectique, tactique). Dans le cadre théorique développé par Adam, on peut dire que c'est au niveau du genre que l'interaction entre les différents paliers d'analyse textuelle (structure compositionnelle, représentation discursive, énonciation et orientation pragmatique) prend sens et devient interprétable.

Or, dans le champ générique que constitue la poésie, les dictionnaires de poétique semblent hésiter entre deux dénominations pour certains types de poèmes, qualifiés tantôt de genres, tantôt de formes fixes. On parle volontiers de genre quand la forme reste relativement libre, ainsi pour l'ode ou l'élégie, et de formes fixes pour le sonnet, la ballade ou le rondeau, qui obéissent à des règles de composition plus strictes. La question centrale est bien de savoir si la structure compositionnelle s'accompagne également de restrictions thématiques et stylistiques et si ces critères peuvent être rapportés à des conditions d'exercice spécifique de l'écriture poétique.

L'épopée apparait ainsi comme un genre, car elle satisfait à la fois à des contraintes thématiques (c'est un récit qui concerne non pas seulement un individu mais une collectivité dont l'identité se trouve renforcée par les valeurs déployées dans le texte, récit centré soit sur une guerre, soit sur les exploits d'un héros confronté à l'adversité), compositionnelles (l'épopée est divisée en chants et c'est un texte long) et stylistiques (l'épopée est écrite en vers amples, sans organisation strophique, dans un style élevé). Qui plus est, l'épopée ressortit à ce que Schaeffer appelle les classes généalogiques : il s'agit d'un genre dont les occurrences se construisent par référence à des modèles inauguraux. Ainsi Anabase ou Vents de SaintJohn Perse, ou Les Indes d'Edouard Glissant, pour ne citer que quelques exemples du $\mathrm{XX}^{\mathrm{e}}$ siècle, doivent être analysés à la lumière des œuvres d'Homère, Virgile et peut-être Camões auxquelles on peut les comparer. 
En ce qui concerne l'ode ou l'élégie, la situation est moins claire car la dénomination renvoie davantage à une tonalité qu'à des caractéristiques formelles. On pourrait parler d'un macro-acte illocutoire de célébration dans le cas de l'ode et de plainte ou de regret dans le cas de l'élégie. Le TLFi définit l'élégie comme un "poème lyrique de facture libre, écrit dans un style simple qui chante les plaintes et les douleurs de l'homme, les amours contrariés, la séparation, la mort » et l'ode comme un " poème lyrique composé de strophes généralement identiques par le nombre et la mesure des vers, consacré à des valeurs importantes, à des sentiments intimes ", indiquant donc des recouvrements thématiques possibles entre les deux sortes de poèmes mais une différence tonale que la suite des articles précise: d'un côté la mélancolie, de l'autre, l'enthousiasme. Mais cette stabilisation tonale antithétique est assez récente et, dans l'Antiquité, l'élégie comme l'ode recouvraient des réalités métriques précises mais des tonalités et des thématiques fort diverses ${ }^{2}$.

On retrouve la même situation, mais amplifiée, pour le sonnet. Inventé au Moyen Âge, le sonnet possède un nom d'origine provençale, mais les premiers exemples en sont italiens. Mis à l'honneur par Pétrarque, il est importé en France au XVI ${ }^{\mathrm{e}}$ siècle : Marot propose six sonnets traduits de Pétrarque et quelques-uns de sa propre inspiration et L'Olive de Du Bellay (1549) est écrit entièrement en sonnets. Mais très vite la stabilité formelle du sonnet (qui admet toutefois de nombreuses variantes) s'accommode d'une très grande variété thématique et pragmatique. Même si Pétrarque a constitué un modèle pour les poètes de la Renaissance, Les Regrets de du Bellay, à dominante argumentative et tonalité bien souvent polémique, et Les Amours de Ronsard, à thématique amoureuse, proposent d'emblée des réappropriations différentes de cette forme (cf. Gendre, 1996). Si une certaine unité peut être trouvée dans les sonnets, elle semble plutôt d'ordre énonciatif: la majorité d'entre eux adoptent en effet une scénographie où un locuteur à la première personne exprime des affects ou des jugements. Néanmoins de nombreux sonnets baroques sont impersonnels et appartiennent à certains égards au genre de la méditation religieuse ou morale. Les sonnets de Heredia et des Parnassiens en général se caractérisent aussi par leur impersonnalité et le déploiement d'une rhétorique du tableau. Ce n'est pas sans raison que Baudelaire écrit :

Tout va bien au sonnet, la bouffonnerie, la galanterie, la passion, la rêverie, la méditation philosophique. (Correspondance, tome I, p.676)

Il serait évidemment possible de rendre compte de cette situation en postulant l'appartenance d'un même texte à plusieurs genres : sonnet et méditation, sonnet et élégie, sonnet et satire, sonnet et tableau, etc. Une telle hybridité générique est défendue pour d'autres textes par Adam et Heidmann dans leurs six propositions sur la généricité (2009 : 11-22). Par ailleurs, la partie consacrée aux plans de texte dans $L a$ linguistique textuelle ne semble pas laisser de doute sur l'identité générique du sonnet.

Adam (2011 : 205) commence par distinguer le plan de texte conventionnel, « fixé par l'état historique», et le plan de texte occasionnel, "inattendu, décalé par rapport à un genre ou à un sous-genre de discours ». Dans ce paragraphe, plan de texte et généricité sont donc étroitement associés, soit que le plan de texte soit dicté par les routines du genre, soit qu'il s'en émancipe.

Un peu plus loin, Adam écrit à propos des plans de texte fixes :

Des plans de texte sont, avec les genres, disponibles dans le système de connaissances des groupes sociaux. Ils permettent de construire (à la production) et de reconstruire (à la lecture ou à l'écoute) l'organisation globale d'un texte prescrite par un genre. » (2011: 205-206)

Il définit ensuite les plans de textes occasionnels comme faisant l'objet d'une procédure empirique «pas à pas » de «(re)construction de [1]a structure [du texte]» (2011:207), procédure plus ou moins guidée par un marquage des « segments textuels » qu'assurent de façon variable les connecteurs, la segmentation en paragraphes ou chapitres, les titres, la répartition des isotopies et des tiroirs verbaux, les changements de topique.

À suivre ces définitions, le sonnet, ainsi que cela est dit explicitement en haut de la p.106, est bien un genre imposant un plan de texte fixe : 2 quatrains + un sizain comprenant 2 tercets typographiques ${ }^{3}$. Pourtant, si l'on compare le sonnet aux autres exemples cités, une dissymétrie apparait: en effet, ni la dissertation, ni la tragédie, ni la plaidoirie n'ont pour seul trait définitoire leur structure compositionnelle. 
On peut également leur attacher un contenu thématique, une représentation discursive et une visée pragmatique qui les différencient d'autres genres et donnent à toutes les occurrences du type, indépendamment des évolutions, un certain air de famille. Il n'en va pas de même pour le sonnet : si l'on tient à définir le sonnet comme un genre, on sera dans le cas assez exceptionnel où, hormis l'appartenance à un champ générique qui est la poésie, rien d'autre ne définira le genre que sa structure. En revanche, si l'on envisage le sonnet comme un plan de texte disponible pour qui veut écrire des poèmes, des perspectives nouvelles s'ouvrent, que je voudrais indiquer à présent.

\section{Potentialités du plan de texte}

Les études sur le sonnet n'ont pas manqué de souligner que la fortune de ce type de poème tient à l'alliance entre la rigueur des contraintes qu'il impose et les richesses sémantiques qui en découlent si on veut bien les exploiter. Le travail célèbre et controversé de Lévi-Strauss et Jakobson sur Les Chats de Baudelaire avait bien mis en valeur le nombre élevé de structures possibles dans le sonnet. Il convient à cet égard de rappeler que, si pour les rimes, le sizain forme un tout et qu'en toute rigueur terminologique, le sonnet ne comporte que trois strophes, la division en tercets typographiques est marquée dès les origines ${ }^{4}$. Alors que, par souci d'économie, on édite les sonnets en blocs compacts, des décrochements indiquent clairement le vers initial de chaque strophe ${ }^{5}$ (c'est au cours du XVII ${ }^{\mathrm{e}}$ siècle que les éditeurs prendront l'habitude de séparer les strophes par des blancs). Le sonnet est donc une structure $8(4+4)+6$ $(3+3)$ qui joue sur le pair et l'impair, l'égalité et la différence.

On peut ainsi opposer les quatrains $\mathrm{AB}$ aux tercets $\mathrm{CD}$, mais on peut aussi opposer $\mathrm{AD}$ (strophes externes) à $\mathrm{BC}$ (strophes internes), opposer entre eux $\mathrm{A}$ et $\mathrm{B}$ d'une part, $\mathrm{C}$ et $\mathrm{D}$ d'autre part, ou bien encore mettre en valeur le potentiel ternaire du sonnet (A/B /CD) en individualisant chacun des 3 groupements. Ces oppositions ou ces différences pourront reposer sur des bases phoniques, rythmiques, morphologiques (verbes vs noms, par exemple), énonciatives (systèmes des marques de personnes et de temps, modalités phrastiques), sémantiques (euphorie vs dysphorie, nature vs culture, animé vs inanimé, ainsi que toutes les oppositions entre isotopies spécifiques que l'on peut imaginer). On trouvera dans l'introduction de Gendre (1996) des citations de traités de poétique des XVI et XVII ${ }^{\mathrm{e}}$ siècles montrant que les auteurs et lecteurs de l'époque étaient parfaitement conscients de ces possibilités. Gendre résume cette idée en disant :

Que ses modèles soient logiques ou dramaturgiques, le sonnet se prête à des schémas de sens préétablis. Même s'ils ne sont pas actualisés par toutes les pièces, tant s'en faut, ces schémas montrent que le dynamisme propre à toute construction poétique est particulièrement mis en relief par les dispositions contraignantes du sonnet. (1996:8)

La subdivision du poème en 4 parties typographiques et 3 parties rimiques permet un grand nombre de parcours tant sur le plan phonique que syntaxique ou sémantique : tantôt se crée un équilibre dynamique où le sizain équivaut aux 2 quatrains, tantôt au contraire les déséquilibres s'accentuent au fil du texte, et, dans l'espace resserré des 14 vers, on peut jouer tour à tour ou simultanément sur la symétrie, les parallélismes et l'asymétrie. Comme le dit Mallarmé ${ }^{6}$, «le sonnet est un grand poème en petit ». Ruwet (1972) a montré avec beaucoup de finesse tout le parti qu'on pouvait tirer de cette structure.

Par ailleurs la brièveté relative du sonnet rend possibles des interactions complexes avec la syntaxe : on peut faire coïncider phrases et strophes, phrases et divisions typographiques, ou, au contraire, jouer sur les enjambements de strophe à strophe, on peut aussi écrire un sonnet composé d'une seule phrase.

Ce qui me semble par ailleurs expliquer la fascination qu'a exercée et qu'exerce encore le sonnet, c'est que, pour contraignante et riche qu'elle soit, cette forme n'est qu'une forme, qui se prête à de multiples investissements thématiques et stylistiques. C'est flagrant lors de sa première floraison à la Renaissance et à l'époque baroque, et, à nouveau, lors de son nouvel essor dans la deuxième moitié du XIXe siècle ${ }^{7}$. Plutôt qu'une énumération fastidieuse des thèmes abordés et des variétés stylistiques observées, il me semble préférable d'indiquer les quelques contraintes textuelles qui découlent de la brièveté de la forme. 
Si le sonnet n'exclut pas le narratif, comme le montre très bien l'analyse que propose Adam du célèbre $\dot{A}$ une passante, il impose généralement un resserrement dans le temps. La dynamique du récit s'en trouve affectée d'une façon qui mériterait certainement de longues investigations : il me semble intuitivement que cela conduit à laisser implicites certaines étapes de la séquence narrative, et à souligner le nœud et le dénouement au détriment des autres propositions.

Cependant, plus souvent qu'un récit, le sonnet nous offre une description d'actions ou une méditation qui, sans prendre la forme d'une séquence canonique, revêtent une dimension argumentative pouvant jouer sur le non-dit ou communiquent au lecteur une intensité affective qui semble être l'enjeu du texte. Le plan de texte, par sa rigueur mais aussi par les possibilités de jeu qu'il offre, se prête à la comparaison (bien visible dans Les Antiquités de Rome, par exemple, et dans maints sonnets baroques comparant la destinée humaine aux nuages ou aux bulles de savon), à l'antithèse, à la gradation, et bien sûr, au retournement, sous la forme rhétorique de la chute. Ce qui importe à l'analyste, c'est donc de mettre en rapport l'organisation périodique, ou plus rarement séquentielle, du texte avec le plan de texte en observant, parmi les potentialités de celui-ci, quelles sont celles que le texte actualise et pourquoi.

Sur le plan énonciatif, le sonnet s'accommode d'un grand éventail de dispositifs énonciatifs mais on peut distinguer deux grandes tendances : un fort engagement du locuteur, tant sur le plan des coordonnées spatio-temporelles que sur le plan des valeurs ou de l'affectivité, ou, au contraire, une impersonnalité qui fait tendre le texte tantôt vers le gnomique, tantôt vers la description rhétorique. Dans les deux cas, cette impersonnalité s'accommode fort bien de lexèmes axiologiques ou affectifs qui proposent au lecteur une vision clairement subjective mais présentée comme universelle.

Pour concrétiser ces remarques générales, je présenterai succinctement trois séries de sonnets : ceux parus en 2011 dans L'Heure présente d'Yves Bonnefoy qui rassemble des textes en prose, des poèmes en vers libres et trois ensembles de sonnets intitulés «Raturer outre », «Soient Amour et Psyché » et «Pour mieux comprendre » $(37 \text { sonnets au total })^{8}$, les poèmes du recueil Vrouz de Valérie Rouzeau, paru en 2012, et le journal en sonnets de Robert Marteau dont les 6 volumes (Liturgie, Louange, Registre, Rites et offrandes, Le temps ordinaire et Écritures) couvrent une période allant de 1987 à 2002. Ces ouvrages mais on pourrait en citer d'autres, notamment $\in$ (Signe d'appartenance) de Jacques Roubaud ou les 41 sonnets irrationnels de Jacques Bens - se réclament de la tradition du sonnet, tout en s'en écartant sur un certain nombre de points. Je les examinerai en montrant tout d'abord le parti qu'ils tirent du plan de texte choisi puis en réfléchissant à leur inscription générique et à son interaction avec le plan de texte.

\section{Trois usages contemporains du plan de texte}

\subsection{Les sonnets de L'heure présente d'Yves Bonnefoy}

Les sonnets des parties 1, 2 et 5 de L'heure présente d'Yves Bonnefoy, recueil qui comporte aussi des poèmes en vers libres et des poèmes en prose, sont les plus proches du plan de texte traditionnel, ainsi que l'indique la note de l'auteur en fin de volume :

Si je n'avais pas adopté ce parti prosodique, quatorze vers distribués en deux quatrains et deux tercets, ces poèmes n'auraient pas existé, ce qui ne serait peut-être pas bien grave, mais je n'aurais pas su ce que quelqu'un en moi avait à me dire.

Les mots, les mots comme tels, autorisés par ce primat de la forme à ce qu'ils ont de réalité sonore propre, ont établi entre eux des rapports que je ne soupçonnais pas. (...) La contrainte aura été une vrille, perçant des niveaux de défense, donnant accès à des souvenirs restés clos si ce n'est pas réprimés. (p.119)

Mais, même s'ils respectent la division en quatrains et tercets, ces sonnets ne sont pas isométriques et ne comportent pas de rimes : les vers oscillent pour la plupart entre 10 et 12 syllabes, on peut très souvent les ramener à des décasyllabes réguliers en ne prononçant pas certains $e$ atones :

Qui est-il, qui s'étonne, qui se demande

S'il doit se reconnaitre dans cette image? 
C'est l'été, vraisemblablement, et un jardin

Où cinq ou six personnes sont réunies.

Et c'était quand, et où, et après quoi ?

Ces gens, qui furent-ils, les uns pour les autres?

Même, s'en souciaient-ils? Indifférents

Comme déjà leur mort leur demandait d'être.

Toutefois celui-ci, qui regarde cet autre, Intimidé, pourtant ! Étrange fleur

Que ce débris d'une photographie!

L'être pousse au hasard des rues. Une herbe pauvre

À lutter entre les façades et le trottoir.

Et ces quelques passants, déjà des ombres. (p.10)

Dans le premier quatrain de «Encore une photographie », si l'on ne tient pas compte des $e$ en septième syllabe, on obtient la métrique : 6-4/6-4/8-4/6-4. Dans le deuxième quatrain, les décasyllabes réguliers des vers 5 et 7 alternent avec les vers 6 et 8 qui peuvent être lus 6-5 . Les tercets sont, quant à eux, composés de décasyllabes réguliers (4-6 ou 6-4) et d'alexandrins (6-6 ou 8-4) si l'on veut bien élider la syllabe finale de façades.

Dans l'ensemble des sonnets, les vers 6-4 alternent avec les 6-5, les 12-syllabes ne sont pas toujours organisés en unités familières (6-6 ou 8-4), certains vers n'ont que 8 syllabes ou moins, de sorte que l'ensemble propose un rapport de fidélité à la tradition qui n'exclut pas une légère distance, où s'entend une maladresse volontaire de qui ne veut pas céder au sortilège de l'harmonie au détriment de la vérité. On pourrait en résumant dire que l'on oscille entre des poèmes à la métrique quasi régulière et d'autres, notamment dans la série «Le nom perdu », où l'hétérométrie et l'irrégularité des coupes font d'autant plus ressortir l'importance du découpage entre strophes qui, seul, assure la filiation avec le sonnet. Le point commun, néanmoins, de tous les poèmes est la relative autonomie de chaque vers, la brièveté des phrases et la soumission de la syntaxe aux limites du vers, même si les enjambements évitent toute rigidité. La forme joue pleinement son rôle de guide : les unités syntaxiques se coulent dans le vers ou enjambent d'un vers sur l'autre mais les strophes correspondent le plus souvent à une unité de sens forte. Les enjambements de quatrains à tercets restent exceptionnels : on ne les trouve que dans la suite "Pour mieux comprendre » (p.54 et 55) où ils correspondent à une forte tension sémantique.

Hormis ces exceptions hautement signifiantes, le plan de texte est exploité dans le rapport de contraste qui s'établit souvent entre les quatrains et les sonnets. Je ne signalerai que les changements les plus fréquents :

- changement de point de vue marqué par un «non» $(p .11,19)$ ou un «toutefois» $(p .10)$, ou une remise en question interrogative de ce qui vient d'être énoncé (p.11, 19, 37);

- déplacement de la narration vers un autre personnage (p.22);

- irruption du je (p. 15, 29) ;

- changement des modalités phrastiques (p.9);

- passage du récit au commentaire (p.16,36), du constatif à l'éventuel (p.32), du passé au présent ou inversement (p.57, 59).

Mais la rupture apportée par le premier tercet peut n'être que temporaire et le second peut reprendre le fil initial : ainsi, à la p.14, le personnage unique désigné par $i l$ est-il présent dans les strophes 1,2 et 4 alors que la strophe 3 évoque "dans d'autres mondes » "deux enfants [qui] s'éloignent sans fin sur une plage ». De même, à la page 28, le premier tercet correspond au discours rapporté des deux personnages et s'oppose aux trois autres strophes décrivant les actions de « elle » et « il ».

On trouve également des cas, plus rares, où le dernier tercet s'oppose à tout ce qui précède, par exemple, p.13 où la parole du «vieil homme », d'abord relatée au discours indirect, passe au discours direct, ou 
p. 20 où on quitte le passé au début du second tercet. On notera aussi que le sonnet de la p.10 cité plus haut oppose les interrogations des quatrains aux exclamatives du premier tercet et le scepticisme à un certain élan, mais que le deuxième tercet apporte à son tour une rupture par ce qu'on peut considérer comme un retour à l'ici-maintenant.

Ce travail sur les contrastes entre strophes refuse cependant toute chute ou tout renversement spectaculaire. On a plutôt affaire à des glissements progressifs, où les énoncés précédents sont passés au crible, rectifiés ou contredits par l'avancée réflexive ou l'insistance du réel, ou bien à des narrations lacunaires, qui se déploient parfois sur plusieurs sonnets dotés d'un titre commun, comme dans « Le nom perdu », «L'écharpe rouge », « Soient Amour et Psyché ». L'unité de chaque texte est assurée par un nombre élevé de reprises lexicales et d'anaphores pronominales d'autant plus importantes que les personnages ne sont souvent désignés que par des pronoms personnels, comme on peut le voir sur ce deuxième exemple intitulé « Donner des noms » :

Elle se penche sur lui, elle murmure :

Veux-tu que nous donnions des noms encore,

Car sais-tu si jamais nous nous reverrons?

Oui, dit-il, je te nomme, hésitation

Qu'a eue ce martinet prenant son vol,

Qu'a-t-il vu qui le tint comme suspendu

Un instant dans le cri de tous ces autres?

Je veux te dénommer pour me souvenir.

Puis il tourne la page. Ce qu'il voit,

C'est cette même jeune femme, souriante,

Elle semble rentrer d'un long voyage.

Comment me nommes-tu ? demande-t-elle,

Inquiète, tristement. Et la nuit tombe,

Ces martinets, une aile immense dans le ciel. (p.27)

Plusieurs sonnets de L'heure présente ont pour origine, si l'on en croit les propos d'Yves Bonnefoy interviewé par Alain Veinstein ${ }^{10}$, des images émanées de l'inconscient, surgies du rêve, d'autres prennent pour point de départ des tableaux ou des photographies. Dans tous les cas, leur unité interne est très forte, les nombreux démonstratifs déictiques rattachent le texte à l'image ou au souvenir dont il émane. Les poèmes allient donc 3 caractéristiques essentielles :

- une relative harmonie entre métrique et syntaxe qui n'est pas systématique mais assez prégnante pour que la forme versifiée paraisse naturelle sans pour autant devenir monotone ;

- une unité interne qui fait de chacun, ou des suites numérotées rassemblant 2, 3 ou 4 textes, un tableau, une description d'actions ou un embryon de récit se suffisant à eux-mêmes, même si leur sens reste généralement très ouvert ;

- un dynamisme de la représentation sémantique ou une évolution des actes illocutoires qui exploitent pleinement le potentiel de la division en strophes.

Si on les compare aux poèmes de Robert Marteau ou de Valérie Rouzeau, ces sonnets sont les seuls à reconduire la séparation traditionnelle entre les strophes. Ils invitent par là à envisager les articulations entre les strophes et les changements qui accompagnent le passage de l'une à l'autre et des quatrains aux tercets comme un élément important de leur représentation sémantique.

\subsection{Les sonnets de Robert Marteau}

Robert Marteau, né en 1925, mort en 2011, est l'auteur d'un journal en sonnets d'une ampleur considérable : six volumes parus aux éditions du Champ Vallon, quatre en préparation. Les volumes parus couvrent les années 1987 à 2002. Chaque sonnet est daté, et si on n'a pas un sonnet par jour, il arrive parfois qu'on ait plusieurs sonnets pour un même jour. J'ai étudié pour les seize années publiées les 
poèmes du mois de mai, mois pour lequel on dispose de 188 sonnets, répartis très irrégulièrement selon les années (de zéro en 1995 à 29 en 1990), avec une moyenne de presque 12 sonnets par an. Ces sonnets se caractérisent par leur compacité (ils ne comportent pas de strophe) et leur caractère non métrique : si chaque vers compte bien 12 syllabes comptées de façon traditionnelle (sans apocope du $e$ atone), aucune césure ou coupe régulière n'est perceptible, à quelques rares exceptions près. Pour les sonnets du mois de mai, les vers césurés 6-6 ne constituent pas plus de 6,5\% du total, soit à peine un vers par sonnet. Par ailleurs Robert Marteau décale systématiquement les fins de vers et les fins de groupes syntaxiques, n'hésitant pas à séparer un pronom clitique sujet et un verbe, un auxiliaire et un participe, un verbe et son complément valentiel, un nom et l'adjectif ou la relative qui le déterminent, une préposition et son régime, voire à scinder en deux un mot composé. On trouvera la plupart de ces ruptures exemplifiées dans le sonnet dans ci-dessous :

Le seringa fleurit à son heure, entre pluie

Du matin et déjeuner de soleil. Il verse

Parmi les plantes la candeur de son parfum ;

Il surprend le jardinier par la subreptice

Expansion de son souffle, l'enneigement

Du bois en mai à qui les sous-bois de muguet

Sont promis. Il aime au nuage offrir l'alcool

Qu'il a extrait de la rosée. En ses parages,

Qu'un corbeau vacille : on voit s'intensifier

Le blanc, tout le buisson s'exalter. Il accorde

Au coucou son encens, enclot la tourterelle

Endormie. On avait oublié qu'il viendrait

Candide et constellé dans la nuit verte qu'il,

Comme un dieu, suscite : immortelle hamadryade.

(Mardi 8 mai 1990, Louange, p. 49)

On est ici face à un choix qui crée deux poèmes en un : celui qu'assure une lecture prosaïque qui ne tient pas compte des fins de vers, et celui que produit une lecture fidèle aux blancs de fins de vers qui dissocie ce que la syntaxe associe et qui réunit dans une entité formelle, le vers, des éléments disparates. Les places initiale et finale acquièrent une importance accrue, en raison de la mise en suspens que crée le blanc de fin de vers. La place médiane, en revanche, n'est plus privilégiée.

Robert Marteau s'est expliqué dans un numéro passionnant de la revue Formules intitulé Le sonnet contemporain $^{11}$ :

Je me suis depuis longtemps interrogé, et toujours m'interroge, sur la fortune du sonnet, me demandant encore aujourd'hui s'il ne concrétisait pas l'espace maximum et la quantité maximale où pouvait sans absence se concentrer l'attention. [...] C'est peut-être en obéissant à cette observation que, peu à peu, soumis à la flexion de la phrase, je me suis mis à recueillir au cours de mes marches, écrivant ainsi avec les pieds, comptant ce qui m'était conté, l'impromptu évènement. [...] Je constatais en même temps que le sonnet était d'abord une grille vide de 168 (12 x 14) cases, qu'il s'agissait de remplir, mais de telle manière que l'artifice menât au naturel. (p.251-252)

Jean-François Rollin, ami de Robert Marteau, assure que le nombre 168 revêtait pour le poète un sens alchimique, car il résultait de la multiplication du 12 des maisons du zodiaque par le double de 7 , nombre des planètes et des métaux. Mais, indépendamment de ces considérations, il importe de voir le déplacement qu'opère Marteau par rapport au plan de texte traditionnel : il abandonne la métrique au profit du seul compte syllabique, qui, certes, est l'élément dominant du système de versification français, mais qui est associé, dans les vers longs, à une organisation interne du vers reposant sur une césure fixe ${ }^{12}$. L'absence concomitante de rime supprime toute anticipation par le lecteur et donne à chaque mot un rôle unique. Un tel choix enferme le texte dans des limites qui, tout à la fois, entravent l'élan propre de la phrase et le font ressortir en le brimant. Ceci est particulièrement net dans les textes qui ne contiennent qu'une ou deux phrases :

L'extraordinaire effet du soleil sur l'eau

Où il se brise comme une assiette en mille 


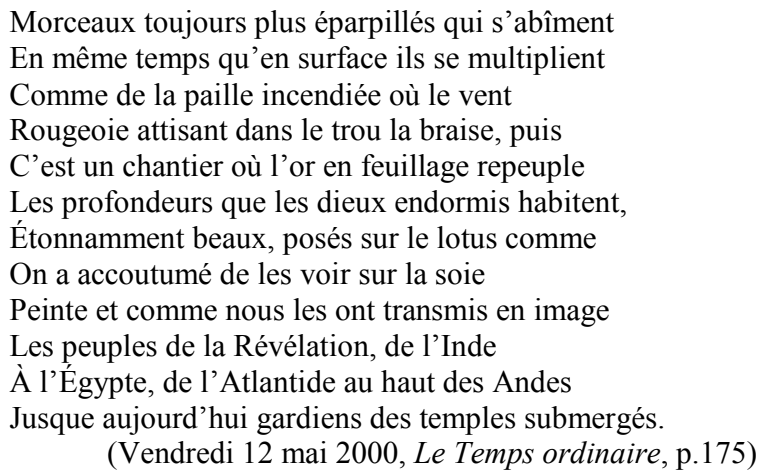

Dans ce poème, on observe cinq relatives, quatre comparaisons (une fois en même temps que et trois fois comme), un puis reliant les deux propositions noyaux, deux groupes détachés apportant des prédications secondes à propos des «dieux endormis » et l'inversion du dernier groupe sujet qui occupe les trois derniers vers : ces choix syntaxiques nous emportent dans un déroulement linéaire où chaque élément entraine le suivant, et où le lecteur est pris dans un vertige créé par l'arrivée constante de nouveaux syntagmes qui font dériver la phrase bien loin de son point de départ. Mais cette logique de la dérive linéaire est contrariée par la mise en vers qui, elle, interrompt le flux, isole des fragments de syntagmes, et nous oblige à refaire une unité constamment brisée. Derrière les déclarations de Marteau qui n'insistent que sur le compte des syllabes et le naturel de ces sonnets prosaïques, il me semble possible, au contraire, de mettre en relief un parti pris stylistique qui permet à l'auteur de conjoindre la fidélité à un plan de texte archétypal et la perception, maintes fois explicitée, d'un monde menacé par la perte d'harmonie et dont la poésie a pour mission de recoller les morceaux éparpillés.

\subsection{Vrouz de Valérie Rouzeau}

Ce livre publié en 2012 à La Table ronde contient 151 poèmes de 14 vers que l'auteure songeait à intituler «autoportraits sonnés avec ou sans moi» (voir la première note p.163) avant que l'acteur Jacques Bonnaffé ne lui suggère Vrouz: titre qui affiche le lien avec l'autobiographie en condensant l'initiale de son prénom et le début de son nom, tout en évoquant le vrombissement d'une machine ou d'un insecte, ou le claquement d'une porte, ou, plus souterrainement, une version joyeuse, plus sonore, de la frousse, en écho à l'inquiétude qui affleure en maints endroits du texte.

Contrairement à ce qu'ont pu écrire certains chroniqueurs, les poèmes ne sont pas écrits en vers libres. Comme le montre très bien Antoine Émaz sur Poezibao ${ }^{13}$, les poèmes sont écrits en vers pairs, de $6,8,10$ ou 12 syllabes, pour peu que l'on ajuste au rythme dominant la prononciation des e atones :

Chaque «sonnet» fait bloc, sans découpage en strophes, sans schéma de rimes obligé, mais avec une contrainte isométrique très forte sur des mètres pairs : $6-8-10-12-14$. Bien entendu, les licences sont fréquentes, diérèses ou compte tenu ou non de l'e muet. Mais le rythme pair est si majoritairement prégnant que la lecture modèle le vers jusqu'à l'entendre régulier. (A. Émaz)

De nombreux poèmes sont isométriques ou bâtis uniquement sur deux mètres, octosyllabes ou alexandrins. Le lien avec la tradition poétique est donc fort, d'autant que les 12 syllabes sont assez souvent césurés 6-6 mais la compacité des poèmes et l'absence de système de rimes (même si les échos sonores sont très nombreux) les éloignent des sonnets usuels, comme on peut le voir ci-dessous :

Tant de jours engloutis de temps à tout jamais

Perdu irrattrapable et je commence demain

Sans angoisse ni black-out je répare dès demain

La carcasse rosserie la carrosse carrosserie

Et le moteur aussi qu'il fasse son meilleur bruit

De montre bien discrète subtile et régulière

À la place de ces boum boum boum de cœur débile

Cet affolement motif euphorie dysphorie 


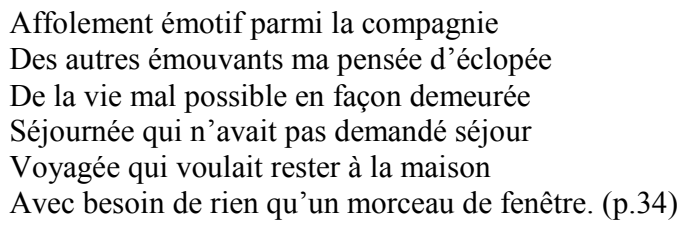

Le poème est écrit entièrement en vers de 12 syllabes avec un certain nombre de $e$ élidés. Il comporte des rimes pauvres en /e/ et /i/ et beaucoup de rimes internes, de jeux de mots, de reprises lexicales. Il s'agit là d'un trait caractéristique du recueil, de même que la coïncidence forte entre fin de vers et fin de syntagme, perturbée néanmoins par des rejets comme ici perdu au vers 2 ou des enjambements du vers 5 au vers 6 , et du vers 9 au vers 10 . On note également un certain flottement de la syntaxe à partir de $m a$ pensée d'éclopée dont le lien avec ce qui précède est assez lâche - à moins qu'il ne s'agisse encore d'un complément de je répare - et l'emploi au passif de séjournée et voyagée qui sont d'ordinaire intransitifs. Les entorses à la syntaxe, les paronomases, et les échos phoniques sont une constante de ces poèmes qui évoluent souvent par association d'idées et de sons mais qui présentent aussi des bouclages assez fréquents, renouant avec la tradition du sonnet :

Dans la multicolore foule de la gare

Les venues les allées des autres des uns

Parmi les cheminots le monde

Je croise un beau garçon aux cheveux blancs

Sa queue de cheval est tenue par une pince

À linge verte comme du printemps doucement

Herbe aussi folle qu'elle en a l'air

Vert d'eau calme pleine de grenouilles prêtes

À crever le plafond de leur chant

La voûte mal céleste de la gare grouillante

De gens plus ou moins encombrants

Plus ou moins encombrés de gens

Allant venant dont ce garçon furtif

Pour qui j'en pince juste en passant. (p.76)

Dans ce poème les vers ont $8,9,10,11$ ou 12 syllabes avec une prédominance des octo- et décasyllabes, mais les rejets et contre-rejets sont très fréquents, mimant la cohue désordonnée de la gare et brouillant le rythme. Le premier et le plus spectaculaire est celui qui éloigne pince de à linge, attirant par là même l'attention sur le détail vestimentaire et préparant l'effet de bouclage humoristique de la fin : le recours à l'homonyme verbal pince permet de clore le poème en renvoyant à ce qui a suscité l'intérêt de la locutrice et que la disjonction pince / à linge soulignait. Le gout de Valérie Rouzeau pour le défigement de collocations ou mots composés se manifeste aussi avec le groupe voûte mal céleste. Le tissage sonore est sensible dans la saturation des voyelles nasales de la fin, l'allitération en $/ 3 /$, la reprise de gens par $j$ 'en et le parallélisme sonore inversé pince/passant ${ }^{14}$. Le poème témoigne par ailleurs d'une capacité à dériver vers des images inattendues à partir d'une notation de couleur et d'une association entre grouillante et grenouilles. Ces procédés qui ne sont pas sans rapport avec ceux de Laforgue ou Prévert donnent à chaque texte une très forte cohésion. On peut donc dire que Valérie Rouzeau garde du sonnet la notion d'un ensemble dont les éléments sont étroitement reliés entre eux non seulement par le sens mais aussi par des schémas prosodiques et des jeux phoniques. Il est d'ailleurs très difficile d'isoler un vers tant les enchainements de vers à vers, avec leurs surprises et leurs cocasseries, constituent une part essentielle de la dynamique du poème. Tout au plus peut-on isoler certains débuts qui commencent par des jeux de mots appuyés ${ }^{15}$ :

Tentatives de tante hâtive pour capter un peu du jour (p.82)

Je voudrais conduire un corail jusqu'à la mer (p.94)

Aussi je est un hôte d'on ne sait qui ni quoi (p.140)

ou observer une construction circulaire où le dernier vers renvoie au premier : 


\begin{abstract}
Avec beaucoup de pluie un rêve de cabane tient / Debout ... Et ton rêve tient debout tout seul même quand tout tremble. (p.60)

Nul jardin no le Japon Annie Girardot ... La belle actrice n'aura pas su pour le Japon (p.61)

Dans les trains anonymes on raconte sa vie ... Et la dame très coiffée descend sans crier gare. (p.75)

Est-ce un travail de sonner comme ça le quotidien ... Et pour combien de temps quel futur seriné. (p.104)

Il y a un gros vase noir vide rempli d'eau de pluie qui attend ... Il y a un gros vase noir vide rempli d'eau de pluie qui m'entend. (p.113)
\end{abstract}

J'ai pu montrer (Monte, à paraitre) que Marteau, lui, singularise moins ses clausules que ses ouvertures. Quant à Bonnefoy, le dernier vers de ses poèmes propose souvent un élargissement mais ne constitue à proprement parler ni un bouclage ni une chute.

De la mise en confrontation de ces trois œuvres, il ressort que le plan de texte qu'est le sonnet ne peut subsister dans son intégrité que si la division en strophes est préservée. Le sonnet accepte mieux l'hétérométrie ou même l'abandon du vers que l'effacement des divisions strophiques. En effet, cette division n'est pas purement formelle, mais s'accompagne d'une différenciation thématique ou énonciative, qui est un des aspects de ce plan de texte, au même titre que la séparation entre exorde, narration, développement et péroraison dans la plaidoirie antique. À cet égard, les sonnets en prose de Roubaud dans $\in$ (Signe d'appartenance) sont davantage des sonnets que les textes compacts de Valérie Rouzeau. On pourrait toutefois imaginer des textes compacts typographiquement mais qui affichent par une structure interne forte leur appartenance au sonnet, en opposant par exemple les huit premiers vers aux six derniers ou en soulignant d'une façon ou d'une autre l'articulation en trois ou quatre parties. Dans le cas de Vrouz, on a plutôt des poèmes de 14 vers où le nombre de vers vaut davantage comme clin d'œil que comme affichage d'un plan de texte. La poéticité réside dans la métrique, l'unité interne de chaque texte, les jeux sur le signifiant et le travail sur le genre que je vais développer à présent. Quant à Marteau, le cas est un peu différent : l'isosyllabisme et les réflexions métapoétiques de l'auteur montrent, à mon sens, que le sonnet n'est pas une pure référence vide. L'écriture de Marteau se bâtit sur la tension qui nait de l'impossible superposition entre l'implacable structure syllabique et le rythme découlant des découpages syntaxiques. Cette tension est au coeur du projet poétique de Marteau. Il s'agit en effet de faire entrer le rituel poétique dans l'ordinaire des jours, comme nous allons le voir à présent.

\title{
4 Retour au(x) genre(s)
}

Ce travail sur le plan de texte qu'est le sonnet doit être accompagné d'un examen des relations génériques qu'entretiennent ces trois œuvres avec des textes littéraires et non littéraires. En effet, si le sonnet en tant que tel ne me parait pas être un genre, mais plutôt un plan de texte devenu emblématique de l'archigenre poésie, les sonnets peuvent se réclamer de genres, poétiques ou non, qui en éclaireront la portée.

Le cas le plus simple dans mon corpus est celui des sonnets de Robert Marteau. En effet, en nommant son œuvre « journal en sonnets », Marteau affiche d'emblée une relation au genre du journal, mais exploite au maximum le contraste entre genre et plan de texte. Le journal est en effet un genre peu contraint, généralement dans une langue plus familière que celle de la norme littéraire du temps. En choisissant d'écrire son journal en sonnets, Marteau affiche son désir de faire de l'écriture journalière un exercice spirituel et scriptural tout à la fois. Il veut que l'écriture soit tenue par la forme et que, par cette forme qui porte en elle référence au zodiaque et aux planètes, le particulier, au lieu de se replier sur l'intime, soit relié au cosmos et aux dieux. Si l'on se penche sur le péritexte, on observe que la tension entre les deux genres est maintenue : en effet, le fait de faire suivre chaque poème de la date de sa composition incluant la mention du jour de la semaine fait référence au journal. Il en va de même pour l'absence de titre des sonnets, qui ne permet pas de les individualiser et pour la table des matières qui ne distingue dans chaque livre que les années. En revanche les titres des recueils - Liturgie, Louange, Registre, Rites et offrandes, Le temps ordinaire, Écritures - sont plus ambigus. Tout d'abord, ils substituent au titre «journal» 
attendu (qui ne figure, lui, que sur la quatrième de couverture due à l'éditeur et dans les textes des critiques) un titre qui pourrait tout à fait être celui d'un recueil de poèmes à orientation religieuse. Mais, si on les compare par exemple aux titres des œuvres de Jean-Claude Renard, autre poète chrétien du $\mathrm{XX}^{\mathrm{e}}$ siècle - Le Dieu de nuit, La Terre du sacre, Le Temps de la transmutation -, on constate que, plus qu'à une expérience religieuse personnelle, ils font allusion à une activité liturgique, rituelle, communautaire. Le seul qui échappe partiellement à ce champ sémantique, Registre, évoque tout à la fois, outre les multiples connotations littéraires et musicales, le registre des baptêmes, des mariages et des décès tenu par les clercs, et le registre tenu par le père de Robert Marteau, selon un parallèle établi par la $4^{\mathrm{e}}$ de couverture :

Chaque soir, sous la lampe, mon père transcrivait à l'encre sur un registre de carton couvert de toile noire ce qu'il avait au cours de la journée inscrit au crayon sur son calepin. C'était tant de fagots ou tant de stères, ou bien le compte de tel ou tel journaliser ou bûcheron à la tâche. [...] je me dis que selon mon mode c'est peut-être un peu ce que je fais aujourd'hui, par les écritures explorant en outre le registre de ma voix.

On observera que le titre Écritures renvoie lui aussi à deux domaines sémantiques différents : les Saintes Écritures, c'est-à-dire la Bible, et la comptabilité d'un commerçant, d'une entreprise. Ainsi, Robert Marteau choisit des titres qui, en raison de leur polysémie, affichent la double appartenance de ces textes : ils ont certes un caractère rituel, servi par une forme, le sonnet, qui est à bien des égards le parangon de la poéticité, et par des choix stylistiques qui font de ces sonnets des textes à part, mais ils affirment également leur lien avec une pratique ordinaire, le registre commercial, la comptabilité quotidienne. On peut noter qu'il existe un verbe commun au journal intime et au livre comptable, c'est le verbe tenir que l'on retrouve aussi dans l'expression lexicalisée tenir compte de. Le sonnet serait ainsi, en tant que plan de texte, ce qui permet le mieux la tenue du compte des jours, avec toutes les variations de sens du déverbal tenue que nous propose le dictionnaire : durée, continuité (une note tenue), manière d'assurer la discipline, l'économie d'un établissement, assiette du cavalier, dignité de la conduite, stabilité. Dès lors, le choix du 12-syllabes et d'une syntaxe en constant désaccord avec les limites de vers se chargent de signification : la poésie apparait comme ce qui permet de restaurer un ordre ancien, de maintenir un équilibre menacé. Mais, de ce point de vue, le faire de Marteau va plus loin que son dire : le texte manifeste une modernité qui résulte de la mise en tension entre cette nostalgie de l'ordre et la conscience des multiples forces qui tirent le monde à hue et à dia. Il ne se résout pas à une unité factice qui évacuerait les contradictions. C'est en ce sens que la forme du sonnet reste opérante alors qu'elle pourrait paraitre en première instance n'être qu'un lointain souvenir.

En ce qui concerne Valérie Rouzeau, l'appartenance générique revendiquée est celle de l'autoportrait, comme l'indique la note 1 p.163 déjà évoquée. Mais il s'agit « d'autoportraits sonnés avec ou sans moi ». Cette expression complexe est un guide de lecture qui attire l'attention sur le rapport au son et au je, tout en indiquant une distance par le jeu de mots sur sonnés et par la pirouette qu'impliquent des autoportraits «avec ou sans moi ». Nous sommes invités à lire chacun des textes comme livrant quelque chose de la personnalité de leur auteur mais sans exhibition, puisqu'aussi bien celui-ci peut s'en absenter pour n'être que regard sur autrui comme dans le texte sur la cavalière qui s'est suicidée (p.86) ou sur la jolie jeune femme qui pianote des textos (p.96Le terme autoportraits invite aussi à un pas de côté par rapport à une poésie lyrique qui se situerait plus dans la confidence, ou dans la plainte. Renvoyant originellement à la peinture, il insiste sur le regard qui extériorise le moi sur une toile, et rend visible ce qui était intérieur. Mais il évoque aussi le genre rhétorique des portraits, très en vogue dans les salons du XVII ${ }^{\mathrm{e}}$ siècle.. Le choix du terme suggère donc à la fois le regard sur soi et le jeu mondain, l'ironie aussi bien que la tendresse.

L'affichage générique de la note incite par ailleurs à lire le sonnet essentiellement comme un plan de texte qui permet de faire sonner les mots. Le participe passé sonnés qui évoque les cloches et autres sonneries réactive l'étymologie du mot sonnet: emprunté à l'italien, qui l'avait lui-même emprunté au français, le mot sonnet est dérivé de son au sens de « air de musique qu'on chante » et désigne d'abord en provençal « une petite chanson». Le sonnet peut ainsi apparaitre comme le plan de texte qui autorise les calembours, les jeux de mots en tout genre, les échos phoniques. On pourrait lire Vrouz en étant attentif à 
l'importance des éléments sonores du quotidien: sonneries de téléphone, roulements des voitures, klaxons, moteurs en tout genre. Le mot sonnet évoque ainsi à la fois un mode de composition du poème et une thématique de l'invasion sonore qui conduit à l'étourdissement, sens fortement actualisé par le participe sonnés qui s'applique volontiers à un boxeur. Choisir le participe plus que le nom, c'est indiquer d'emblée qu'il ne s'agira pas de sonnets réguliers, que la démarche titubante y sera autorisée et que les rimes internes et allitérations seront là pour permettre des glissements de sens plus que pour assurer une cohésion impeccable.

Mais à lire le recueil, l'indication générique fournie par cette note doit être relativisée : plus que des portraits, auto ou non, ces textes apparaissent comme des tableaux, si l'on veut garder le rapport à la peinture, ou des croquis, pour indiquer leur aspect de scènes prises sur le vif, notées dans un carnet, au fil des déplacements en métro, en train, dans les magasins ou dans les rues des villes. Pour la plupart, ces scènes décrivent un moment ou un lieu qui donne au poème son atmosphère. Parfois, un évènement plus spécifique, mais de peu d'importance, prend place dans le cadre spatial ou temporel ainsi dessiné : rencontre, achat, phrase ou attitude saisie au vol, souvenir d'une citation. Quelques scènes sont présentées comme itératives, résumant un grand nombre de moments identiques. Si donc un autoportrait se dessine, il est plutôt indirect et résulte de la mise en relation de ces différents poèmes avec l'énonciatrice qui s'en donne comme la source. L'unique autoportrait au sens strict est le poème d'ouverture, mais il a ceci de remarquable que la seule propriété positive reconnue à l'énonciatrice par elle-même, c'est son penchant pour l'écriture :

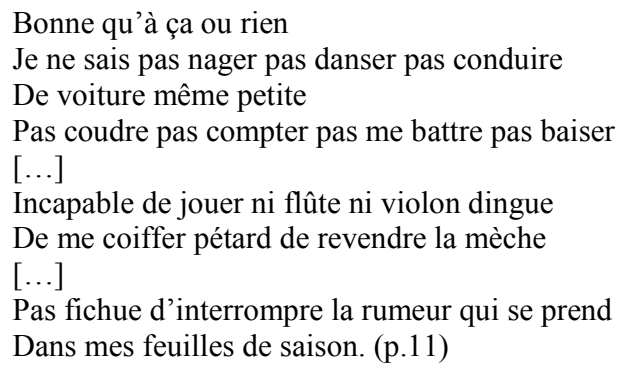

Outre l'affichage d'un éthos d'autodérision que les poèmes suivants confirmeront, ce poème propose dans ses derniers vers une autre indication générique, celle de « feuilles de saison » et une scène énonciative où l'énonciatrice apparait comme celle qui capte «la rumeur». Ces termes qui renvoient au monde des journaux permettent dès lors de lire les textes de Vrouz comme la forme prise par le billet médiatique quand il devient poème. Si on compare ces billets à ceux qu'on trouve généralement dans les journaux et qu'a analysés Sylvie Durrer (2001), on est frappé par leurs points communs: le ton volontiers humoristique, l'aspect apparemment insignifiant des scènes rapportées mais le désir de dresser malgré tout, par petites touches, un portrait de la vie moderne, saisie notamment par ses travers. Les différences se trouvent, bien entendu, du côté du travail sur le langage, qui passe par la contrainte des 14 vers et d'une métrique certes assouplie mais malgré tout présente, par les échos phoniques et les défigements et par les bouleversements apportés à la syntaxe, notamment en raison du recours fréquent aux juxtapositions. Le travail de détournement qui opère dans de nombreux textes prend alors tout son sens : la critique du discours standardisé des affiches, des publicités, des étiquettes de produits alimentaires ou cosmétiques, ou des textes administratifs passe par des citations recontextualisées ou des parodies qui font ressortir la vacuité ou le mensonge de ces discours. Le livre leur oppose des paroles ou des situations qui, en évoquant le point de vue des victimes du système économique, démentent l'enthousiasme des slogans. Si autoportrait il y a, il s'agit donc d'un autoportrait de notre société, qui parfois reprend et renforce la tonalité satirique du billet de presse, mais d'autres fois fait entendre des voix et des émotions minuscules ignorées des médias.

Les sonnets de Bonnefoy, eux, frappent par leur diversité, qui rend difficile la perception d'un genre. Certains titres indiquent le point de départ du poème («Une photographie», «Un souvenir », « Une page de Robert Antelme »), d'autres attirent l'attention sur un détail significatif qui acquiert une valeur symbolique ( L'écharpe rouge », «Branches basses », «Il s'éloigne », «Il gagne la haute mer »), la 
plupart désignent le thème du poème ou en résument l'essentiel (« Le nom perdu », « Le pianiste », « Donner des noms », « Soient Amour et Psyché », « La beauté »). Quelques-uns ont une dimension narrative, ainsi « Le nom perdu » qui déploie sur trois sonnets l'histoire d'un vieil homme assis par terre qui tend à un adolescent un livre, ou «Soient Amour et Psyché », qui, en quatre sonnets, retrace la légende de Psyché racontée par Apulée. Mais dans «Le nom perdu », il est difficile d'assigner un sens univoque aux actions racontées par le locuteur témoin et donc de construire une véritable intrigue qui opposerait un état initial et un état final. Quant à "Soient Amour et Psyché », le mythe constitue l'arrière-plan mais il fait l'objet d'un commentaire plus que d'une véritable narration qui en ferait ressortir la structure et la morale. Plusieurs textes sont, semble-t-il, des commentaires de tableaux et d'autres émanent d'images saisies en rêve, comme le dit Bonnefoy dans l'entretien déjà cité. Cette valeur commentative dote les textes d'une dimension argumentative, qui, certes, ne va pas jusqu'à la construction d'une séquence argumentative où se distingueraient nettement deux thèses opposées mais qui incite au débat, à la réflexion : en témoignent le renversement opéré entre les quatrains et les tercets, marqué par exemple par un « non », le dialogue intertextuel avec Baudelaire (p.12 et 58) qui conteste les positions du devancier les nombreuses interrogations, les appels au lecteur ou l'interpellation à deux reprises de Psyché (p.37 et 53).. En raison de cette forte dimension argumentative, il me semble possible de voir dans ces poèmes des méditations. La méditation est un genre du discours religieux, philosophique mais aussi littéraire, comme l'attestent de nombreux poèmes baroques ainsi intitulés et bien sûr les Méditations poétiques de Lamartine :

La méditation, dans son acception religieuse, est traditionnellement un préliminaire à l'oraison, fondé sur l'approfondissement du sens d'une formule, le plus souvent biblique, ou d'un signe (tête de mort des Vanités par exemple). [...] De façon moins stricte, «méditation » désigne ensuite un état de concentration intérieure, et des textes qui cherchent l'intimité de l'être en se fixanr sur un objet ou un thème. (Fragonard : 2002).

Les thèmes traités par Bonnefoy sont proches de ceux qu'abordaient ses devanciers: réflexion sur le sens de l'existence, sur la mort, le temps, le rêve et l'illusion. Bonnefoy y ajoute deux thématiques qui lui sont chères, la vertu ou le leurre des images, le pouvoir ou l'impuissance de la parole. Le point de départ textuel (l'histoire de Psyché), visuel (tableau, photographie, image onirique) ou sonore (un mot, une phrase entendus et ruminés) évoque la technique même de la méditation religieuse et les répétitions des mêmes mots ou expressions évoquent cet «art de la redite et de l'écho » caractéristique de ce genre selon Fragonard. L'importance des formes du discours (deuxième personne, interrogations, souhaits) s'inscrit également dans cette filiation puisqu'à l'origine, la méditation est un dialogue entre le croyant et Dieu, dialogue remplacé ici par un examen intérieur et une interpellation du lecteur.

Dire que les sonnets de L'heure présente s'inscrivent dans le genre de la méditation ne signifie pas que Bonnefoy ait explicitement fait ce rapprochement. En effet, ses poètes de référence (Baudelaire, Rimbaud, Mallarmé, Yeats, Celan) n'utilisent pas le mot pour définir tout ou partie de leur œuvre. Inversement, l'emploi du nom pour désigner l'œuvre n'a qu'un rôle indicatif et doit être confronté à d'autres caractéristiques : les Méditations poétiques de Lamartine sont bien éloignées des méditations des $\mathrm{XVI}^{\mathrm{e}}-\mathrm{XVII}{ }^{\mathrm{e}}$ siècles et sont plutôt à considérer du point de vue générique comme des élégies. Envisager L'heure présente sous l'angle de la méditation, c'est user d'une clé de lecture qui permet de penser la production poétique sur une longue durée indépendamment des patrons formels auxquels la description veut parfois la réduire : l'usage d'un terme fédérateur pour désigner des textes qui, à partir d'un point de départ sensoriel ou d'une autre création esthétique, réfléchissent sur la destinée humaine permet de discerner ce qui les rapproche et les distingue les uns des autres. C'est ainsi que, contrairement aux poèmes lamartiniens que $\mathrm{j}$ 'ai rapprochés de l'élégie, on peut identifier dans les sonnets de Bonnefoy un je très peu individualisé qui correspond bien à ce genre où l'intimité n'est exposée que parce qu'elle rejoint les questions de chacun, sans rien sacrifier à l'anecdote. Par ailleurs le mot méditation conduit aussi à mieux penser la tension entre la forme du sonnet et la visée des poèmes : si les baroques ont beaucoup écrit en sonnets, leurs méditations sont parfois plus longues, prenant la forme de stances, de cantiques, de paraphrases de psaumes, composés de sizains ou dizains aux schémas métriques variés. Le sonnet impose une réduction drastique à la méditation et conduit également à s'attacher davantage aux mots eux-mêmes, à exploiter au maximum leurs relations et leur place, ce que Bonnefoy ne manque pas de souligner. De ce 
point de vue, le renoncement de Bonnefoy à la chute (qu'il connait pourtant fort bien en tant que traducteur des sonnets de Shakespeare) est très éclairant de son projet : le poème, comme je l'ai dit plus haut, propose, au lieu d'un retournement qui serait propice à la conversion mais clorait la réflexion, une interrogation qui reste ouverte et qui se déplace au fil des strophes.

\section{Conclusion}

Il y a, me semble-t-il, tout intérêt à envisager le sonnet strictement comme un plan de texte : cela incite le linguiste à examiner avec attention les relations qui s'établissent entre ses différentes strophes, comme l'ont illustré de célèbres études, et pourrait déboucher sur une typologie plus rigoureuse des diverses structures et des différents enchainements possibles. Cela permet également d'analyser les sonnets compacts tels que ceux de Rouzeau ou Marteau en prenant l'exacte mesure de la transgression qu'ils opèrent et des modes d'avancée du texte qu'ils instaurent. Il est ensuite possible de confronter ce plan compositionnel aux modèles génériques qui travaillent le recueil de poèmes et d'évaluer la tension ou au contraire la convergence entre les attendus des genres et les normes internes déployées par le type de sonnet choisi. Dans ce rapport aux genres, le sonnet va alors jouer comme parangon de la poéticité : il n'est pas un genre en tant que tel mais un signe de la poésie, et son emploi permet de reconfigurer des genres non littéraires en les faisant basculer vers ce qui apparait dans notre culture comme la quintessence de la littérature en tant qu'art verbal. C'est ainsi que, dans mon corpus, les sonnets de Robert Marteau exploitent au maximum la tension entre l'écriture ordinaire du journal, essentiellement linéaire, et la totalité que constitue le sonnet, en accord avec sa vision d'un monde désenchanté et disloqué mais susceptible d'être remembré par la poésie. Ceux de Valérie Rouzeau proposent une version poétique et critique du billet journalistique tout en renouvelant le lyrisme par la construction d'un éthos distancié. Le recours à une forme aussi exemplaire de la poéticité pourrait être interprété comme une forme de dérision. Mais, outre que les poètes à la posture la plus critique tels que Corbière, Laforgue ou Jacob, usent peu du sonnet, la compacité choisie par Rouzeau établit une référence lâche au modèle qui fonctionne plutôt comme le signal d'une certaine confiance dans la capacité de la poésie à dire les émotions du quotidien en critiquant le prêt-à-porter des discours courants. Quant aux poèmes de Bonnefoy, ils assument pleinement l'héritage de la poésie conçue comme une réflexion sur le sens de l'existence et ils utilisent au mieux les potentialités compositionnelles de la division strophique pour faire tenir en 14 vers des méditations bien souvent marquées par des retournements argumentatifs et des changements de points de vue. Les trois auteurs, par le recours à un plan de texte ancré dans la mémoire collective, inscrivent sans ambages leurs poèmes dans l'archigenre : il leur est dès lors loisible de réinterpréter sur le mode poétique des genres non spécifiquement littéraires (journal, registre, méditation, billet) et, par les torsions qu'ils leur imposent, de manifester ainsi le pouvoir toujours vivant de la parole poétique.

\section{Corpus}

Bonnefoy, Y. (2011). L'Heure présente. Paris : Mercure de France.

Marteau, R. (1992). Liturgie. Seyssel : Champ Vallon.

Marteau, R. (1996). Louange. Seyssel : Champ Vallon.

Marteau, R. (1999). Registre. Seyssel : Champ Vallon.

Marteau, R. (1999). Rites et offrandes. Seyssel : Champ Vallon.

Marteau, R. (2009). Le Temps ordinaire. Seyssel : Champ Vallon.

Marteau, R. (2012). Écritures. Seyssel : Champ Vallon.

Rouzeau, V. (2012). Vrouz. Paris : La Table ronde. 


\section{Références bibliographiques}

Adam, J.-M. (2011, $3^{\mathrm{e}}$ éd.). La linguistique textuelle : Introduction à l'analyse textuelle des discours. Paris : Armand Colin.

Adam J.-M. et Heidmann U. (2009). Le Texte littéraire. Louvain-la-Neuve : Academia Bruylant.

Bakhtine, M. (1984, trad. fr.). Esthétique de la création verbale. Paris : Gallimard.

Baudelaire, Ch. (1973). Correspondance, tome I, 1832-1860, Pichois Cl. (éd.). Paris : Gallimard, coll. « La Pléiade».

Calame, Cl. (1998). La poésie lyrique grecque, un genre inexistant ? Littérature, 111, 87-110.

Cornulier de B. (1995). Art poëtique. Notions et problèmes de métrique. Lyon : PUL, coll. «IUFM ».

Durrer S. (2001). De quelques affinités génériques du billet, Semen, 13, [en ligne] consulté le 17 novembre 2013. URL : http://semen.revues.org/2600

Durand, P. (2008). Avatars de la forme sonnet au XIX ${ }^{\mathrm{e}}$ siècle. Formules, 12, 257-280. Consulté en ligne le 22/10/2013/ http://www.formules.net/revue/12/index.htm.

Fragonard, M-M. (2002). Article «Méditation», Dictionnaire du littéraire, Aron P., Saint-Jacques D. et Viala A. (dir.). Paris : PUF.

Gendre, A. (1996). Évolution du sonnet français. Paris : PUF, coll. « Perspectives littéraires ».

Gouvard, J.-M. (1999). La Versification. Paris : PUF, coll. « Premier cycle ».

Mallarmé, S. (1995). Correspondance complète, 1862-1871 suivi de Lettres sur la poésie, 1872-1898, Marchal B. (éd.). Paris : Gallimard.

Monte, M. (à paraitre). La phrase et le vers dans les sonnets de Robert Marteau : dissociation et reconfiguration. In Bédouret-Larraburu S. et Casanova J.-Y (dir.). Robert Marteau. Actes du colloqued'octobre 2013 à Pau, , La Licorne. Rennes : PUR.

Rastier, F. (1989). Sens et textualité. Paris : Hachette.

Ruwet, N. (1972). Langage, musique, poésie. Paris : Le Seuil, coll. « Poétique ».

Schaeffer, J.-M. (1996). De deux facteurs institutionnels de la différenciation générique. In Rastier, F. (éd.), Textes et sens, Paris : Didier éruditions, 49-66.

\footnotetext{
${ }^{1}$ Je remercie Guillaume Berthon, de l’Université de Toulon, pour sa relecture attentive qui m’a épargné des erreurs.

${ }^{2}$ L'élégie antique est définie par le TLFi comme un «poème aux sujets variés mais le plus souvent mélancoliques, composé de distiques élégiaques », et l'ode antique comme un "poème lyrique destiné à être chanté ou accompagné de musique, composé d'une strophe, d'une antistrophe et d'une épode ». Mais cette définition basée sur des énoncés produits aux $\mathrm{XIX}^{\mathrm{e}}$ et $\mathrm{XX}^{\mathrm{e}}$ siècles néglige la dimension rituelle de la poésie grecque, analysée de façon très éclairante par Calame 1998.

${ }^{3}$ Se référant aux divers schémas de rimes possibles, Gendre (1996:17) parle de forme « semi-fixe ».

${ }^{4}$ En France du moins, puisque les poètes élisabéthains détachent le dernier distique.

${ }^{5} \mathrm{~L}$ 'accès en ligne aux éditions originales sur Gallica permet de le vérifier aisément.

${ }^{6}$ Correspondance, Gallimard, 1959, p.32.

${ }^{7}$ Sur le rapport des poètes du XIX ${ }^{\mathrm{e}}$ siècle au sonnet, on pourra lire l'excellent article de Pascal Durand (2008).

${ }^{8}$ Les deux premiers avaient déjà paru en 2009 aux éditions Galilée sous le titre commun «Raturer outre », le troisième était inédit.

${ }^{9}$ Cette lecture parait préférable à la lecture 5-5 avec apocope d'un $e$, car elle part du décasyllabe régulier pour le gauchir un peu, au lieu d'instaurer une autre césure.

${ }_{10}^{10}$ Du jour au lendemain du 29 octobre 2011, radio France Culture.

${ }^{11}$ Formules est consultable en ligne www.formules.net. Le numéro 12 intitulé Le sonnet contemporain rassemble des textes de poètes et les actes d'un colloque organisé à Poitiers par Dominique Moncond'huy.

${ }^{12}$ Je renvoie pour plus de précisions aux travaux de Benoît de Cornulier (1995) et Jean-Michel Gouvard (1999).

${ }_{13} \mathrm{http} / /$ poezibao.typepad.com/poezibao/2012/03/vrouz-de-val\%C3\%A9rie-rouzeau-par-antoine-emaz.html

${ }_{14}$ Je parle de parallélisme inversé car on a consonne /p/ + voyelle nasale tonique + consonne /s/ + voyelle orale atone dans le premier verbe et consonne $/ \mathrm{p} /+$ voyelle orale atone + consonne $/ \mathrm{s} /+$ voyelle nasale tonique dans le second.
} 
${ }^{15}$ Dans les exemples cités, on a successivement une homonymie in praesentia façon Desnos ou Bobby Lapointe, une homonymie in absentia sur corail (animal marin et sorte de train) et une paronymie (hôte/autre) avec écho du célèbre énoncé de Rimbaud. 